\title{
Evaluation of Dosimetric Effect of Millennium and High Definition Multi Leaf Collimator Using 6 MV Photon Beams
}

\author{
Aswathi Raj ${ }^{1,2 *}$, D Khanna ${ }^{1}$, Hridya V T ${ }^{1,2}$, Sathish Padmanabhan ${ }^{2}$, P Mohandass ${ }^{3}$
}

\begin{abstract}
Purpose: The present study aims to compare different dosimetric parameters from field sizes defined by secondary and tertiary collimators. A comparison has been drawn between two types of Multi Leaf Collimator (MLC) designs. Materials and Methods: The measurements were obtained using Millennium MLC (Mi-MLC) from Varian Unique ${ }^{\mathrm{TM}}$ linear accelerator (LINAC-1) and compared with measurements from Varian Truebeam ${ }^{\mathrm{TM}}$ linear accelerator (LINAC-2) using High Definition MLC (HD-MLC). Dosimetric analysis included percentage depth dose (PDD), cross profile, dosimetric leaf gap (DLG) and scatter factor (SF) that were taken for different field sizes defined by both the MLC design and jaw. For beam data measurement PTW Radiation field analyse (RFA) was utilized. Results: When the surface dose for MLC field for linac 1 and linac 2 were compared with jaws they were found to be on the higher side that is $2.8 \%$ to $4.9 \%$ and $2.2 \%$ to $3.6 \%$ respectively. The SF was found to vary from $-3.2 \%$ to $0.73 \%$ for LINAC- 1 with Mi-MLC when compared with jaws. Similarly, the SF variation from $-2.4 \%$ to $1.1 \%$ was observed for LINAC-2 with HD-MLC as compared with jaw. Larger field sizes gave increased SF while smaller field sizes showed the opposite for HD-MLC. The penumbra was found to be less in HD-MLC as compared to Mi-MLC. Similarly, DLG was found to reduce by $0.056 \mathrm{~mm}$ in Mi-MLC when compared with HD-MLC. The results of symmetry and flatness were seen within the limits for both MLC designs. Conclusion: It can be concluded from the results that both the MLC designs have merits and demerits that are based on their effectiveness and clinical use. However, higher surface dose was found in HD-MLC in contrast to Mi-MLC.
\end{abstract}

Keywords: Millennium MLC- high definition MLC- surface dose- scatter factor- penumbra

Asian Pac J Cancer Prev, 22 (12), 3883- 3888

\section{Introduction}

Multi-leaf collimators (MLC) are moveable leaves that can shape as per irregular tumour in 3 Dimensional Conformal Radiotherapy (3D-CRT) and modulate the intensity of the beam in Intensity Modulated Radiotherapy (IMRT) thereby reducing the normal tissue complications. These treatment techniques have required complex beam arrangements with irregularly shaped MLC fields. The dosimetric parameters have an impact on the dose distribution especially in small fields defined by MLC. Therefore, it is necessary to perform different dosimetric verification of different collimators before any clinical implementation. Commercially, there are different collimator/MLC designs are available from various linac vendors. If the Radiotherapy department have more than one LINAC, the physicist should know the difference in the dose distribution with different LINAC head or with different MLCs even though it cannot be matched dosimetrically

Many studies investigated dosimetric parameters by comparing different collimator designs from different vendors manufactured linac systems. Mohan et al., (2008) studied characteristics of Varian $2300 \mathrm{CD}$ Linac with Millennium 120 MLC for 6 and 18 MV photon beams using three field-defining methods namely, jaw only, MLC only and

MLC + jaw. Percentage depth dose, beam profile, surface dose and dose in the build-up region were used for comparison. The study results suggested that the MLC system with $6 \mathrm{MV}$ and $15 \mathrm{MV}$ are same in all aspects except the surface dose, penumbra, dose in the buildup region and width of $50 \%$ isodose level. Similarly, Kehwar et al., (2006) evaluated dosimetric characteristics of Varian Clinac $2300 \mathrm{CD}$ linac having 80 Millennium MLC (Mi-MLC) systems. The results of this study suggested that MLC play an important role in dosimetric characteristics of 3D CRT, IMRT and beam data collection.

Huq et al., (2002) compared dosimetric characteristics of three MLC systems from Elekta ${ }^{\mathrm{TM}}$, Varian ${ }^{\circledR}$ and Simens ${ }^{\circledR}$ linacs having $1.0 \mathrm{~cm}$ leaf width at isocenter using $6 \mathrm{MV}$ photon beam. Their results revealed that no perfect 
MLC system that could be recommended, rather each one has unique advantages and disadvantages that should be weighed with comfort, ease and cost-effectiveness for clinical use. Asnaashari et al., (2012) compared dosimetric parameter of two MLC systems namely, Beam Modulator (BM) and Radionics micro MLC (m-MLC) from Elekta synergy linac using measurements and Monte Carlo (MC) simulations. Both m-MLC and BM can be used effectively based on dosimetric characteristics for stereotactic radiosurgery (SRS) and radiotherapy (SRT), although the former showed slightly sharper dose penumbra, particularly in the leaf-end direction.

A study by Godson et al., (2016) performed a dosimetric comparison of output factor between BrainLab circular cones, Brain Lab m-MLC and Mi-MLC using ion chamber and diode detector. The result concluded that the orientation of the detector and the position of jaws could influence the output factors significantly in small fields. Based on the literature review, very limited information is available on the comparison of dosimetric parameters of different MLC design/models from the same vendor or different vendors. To fill these lacunae, the present study focused on the comparison of the various dosimetric parameters of two types of MLC i.e., Millennium 120 MLC and High Definition 120 MLC (HD-MLC) from $\operatorname{Varian}^{\circledR}$ Linac systems.

\section{Materials and Methods}

The measurements were performed using $6 \mathrm{MV}$ photon beam on both Varian Unique Power 120 Millennium MLC (Mi-MLC) and Varian True Beam ${ }^{\text {TM }}$ STx 120 High definition MLC (HD-MLC). PTW MP3 water phantom along with $0.125 \mathrm{cc}$ chambers was used for measurements.

\section{Millennium 120 MLC model}

Varian Unique power linac has Millennium 120 MLC (60 pairs) which are attached to the gantry head as tertiary collimators with $0.5 \mathrm{~cm}$ leaf resolution at isocenter. MiMLC is capable of generating maximum static field size of $40 \mathrm{~cm}$ x $40 \mathrm{~cm}$, static aperture field size of $20 \mathrm{~cm}$ x $40 \mathrm{~cm}$ and IMRT field size of $34 \mathrm{~cm} \mathrm{x} 40 \mathrm{~cm}$. The MLC leaves can retract and extend a maximum distance of $20.1 \mathrm{~cm}$ and $20 \mathrm{~cm}$ from the centre line. The maximum displacement between adjacent leaf ends at a single carriage position is $15 \mathrm{~cm}$. Mi-MLC leaves have a maximum leaf speed and carriage speed of 0 to $2.5 \mathrm{~cm} / \mathrm{sec}$ and 0 to $1.2 \mathrm{~cm} / \mathrm{sec}$. The leaf penumbra is defined as the $20-80 \%$ leaf end measured using $10 \mathrm{~cm}$ x $10 \mathrm{~cm}$ field size at depth of maximum dose with $100 \mathrm{~cm}$ source to surface distance(SSD) which is less than $4 \mathrm{~mm}$ for $6 \mathrm{MV}$ beam. Mi-MLC operates in static, dynamic and conformal arc modes such as step and shoots and sliding window IMRT delivery.

\section{High Definition 120 MLC}

HD 120 MLC has 60 pairs with $2.5 \mathrm{~mm}$ leaf width in the central region for $8.0 \mathrm{~cm}$ and $5 \mathrm{~mm}$ leaf width in the periphery. It is capable of generating a maximum field length of $22 \mathrm{~cm}$. The maximum static field size offered by HDMLC is $40 \mathrm{~cm} \times 22 \mathrm{~cm}$, maximum static aperture field size of $30 \mathrm{~cm} \times 22 \mathrm{~cm}$, and a maximum IMRT field size of $32 \mathrm{~cm} \times 22 \mathrm{~cm}$.

The maximum retracted and extended leaf position is $20.1 \mathrm{~cm}$ and $20 \mathrm{~cm}$ and maximum displacement between adjacent leaf end at a single carriage position is $15 \mathrm{~cm}$ with a leaf and carriage speed of 0 to $2.5 \mathrm{~cm} / \mathrm{sec}$ and 0 to $1.2 \mathrm{~cm} / \mathrm{sec}$. The leaf penumbra for HDMLC is less than $3.5 \mathrm{~mm}$ for $6 \mathrm{MV}$ beams for field size $10 \mathrm{~cm} \times 10 \mathrm{~cm}$ at depth of maximum dose and SSD $100 \mathrm{~cm}$.

\section{Dosimetric system}

Semiflex ionization chamber of volume $0.125 \mathrm{cc}$ with UNIDOS $^{\circledR}$ E (PTW, Germany) electrometer was used for measurement. The chamber detectors are vented cylindrical ionization chamber which operates up to \pm 400 $\mathrm{V}$. It has a dimension of the sensitive volume of radius $2.75 \mathrm{~mm}$ and length $6.5 \mathrm{~mm}$ and a total wall area density of $78 \mathrm{mg} / \mathrm{cm} 2$. The central electrode is Al 99.98 with a diameter of $1.1 \mathrm{~mm}$. The chamber is mounted on the large size motorized PTW MP3 3D water phantom with the scanning range of $500 \times 500 \times 408 \mathrm{~mm}^{3}$. The scans were performed using therapy beam analyser MEPHYSTO $\mathrm{mc}^{2}$ V3.2 software.

\section{Dosimetric parameters used for analysis}

The measurements were performed for dosimetric comparison between two systems for different field sizes defined by both jaws and MLC. Various dosimetric parameters such as PDD, beam profile and output factors were measured. The output factors were measured for square field sizes defined by jaws alone and MLC alone. Besides surface dose, dose at $10 \mathrm{~cm}$ depth, depth of $80 \%$ dose and depth of $90 \%$, penumbra total scatter factor, and Dosimetric Leaf Gap (DLG) were evaluated for comparison.

\section{Percentage depth dose and Profiles}

The central axis PDD and beam profile measurements were performed using PTW RFA system along with semiflex chambers of volume $0.125 \mathrm{cc}$. The Radiation Field Analyzer (RFA) was aligned with the crosshair of the linac machine followed by a dry run to for clearance and accurate alignment of the chamber. The centre check was verified to ensure the position of the chamber at isocenter. Keeping Source to Surface Distance (SSD) at $100 \mathrm{~cm}$ PDD and profile measurements were carried out for $2 \times 2 \mathrm{~cm}^{2}, 5 \times 5 \mathrm{~cm}^{2}, 10 \times 10 \mathrm{~cm}^{2}$, and $20 \times 20 \mathrm{~cm} 2$ field sizes defined by jaws alone and MLC alone. Cross profile measurements were performed for $10 \mathrm{~cm} \mathrm{x} 10 \mathrm{~cm}$ field size at $1.5 \mathrm{~cm}$ and $10 \mathrm{~cm}$ depth for comparison.

\section{Output factors}

The output factor measurements were also performed in the RFA system with $0.125 \mathrm{cc}$ semi-flex chamber at $10 \mathrm{~cm}$ depth with SSD $100 \mathrm{~cm}$. The output factors were measured for field sizes $2 \times 2 \mathrm{~cm}^{2}, 5 \times 5 \mathrm{~cm}^{2}, 10 \times 10 \mathrm{~cm}^{2}$, and $20 \times 20 \mathrm{~cm}^{2}$. The output factors were measured for field sizes defined by MLC alone and jaws alone. The measurements were carried out in the same manner in both LINACs to avoid discrepancies. The field sizes were limited to $20 \times 20 \mathrm{~cm}^{2}$ as the maximum field size defined by HD-MLC is $22 \mathrm{X} 40 \mathrm{~cm}^{2}$. The scatter factor is defined 
as the ratio of dose for a particular field size to dose for reference field size $\left(10 \times 10 \mathrm{~cm}^{2}\right)$ were measured in a water phantom at $10 \mathrm{~cm}$ depth for different field sizes defined by jaws and MLC.

\section{Dosimetric leaf gap}

The DLG accounts for the difference between the nominal leaf positions and the radiological leaf positions to incorporate the round-leaf-end effect in dose calculations. It also incorporates the minimal physical gap between leaves to prevent the collision. The DLG measurements were performed by the sweeping gap method. The measurements were taken by placing farmer chamber at $5 \mathrm{~cm}$ with Unidose E, $100 \mathrm{MU}, 10 \times 10 \mathrm{~cm}^{2}$, field size, $95 \mathrm{~cm}$ SSD, with A bank MLC closed, B bank MLC closed, and dynamic sweeping gaps of 2, 4, 6, 10, $16,20 \mathrm{~mm}$.

The net charge without radiation transmission at sweeping gap g was calculated using the following equation

$$
\text { Qnet }(\mathrm{g})=\mathrm{Q}(\mathrm{g})-\mathrm{QT}(1-(\mathrm{G} / 120))
$$

Where $\mathrm{Q}(\mathrm{g})$ was the total charge collected for the beam with the gap $\mathrm{g}(\mathrm{mm})$, QT was the average transmitted charge when the detector was blocked by MLC and 120 was the sweeping gap movement range. Q net was plotted and fitted as a function of $G$ and the linear equation was generated. The intercept value is the DLG value which was the value of $\mathrm{G}$ when the charge becomes zero. The same measurement was done with collimator at 00 and 900 and the average value was noted down. The same method was performed for both the linac systems and tabulated in Table 8. The MLC transmission factor was calculated by dividing the average transmitted charge by open field charge.

\section{Results}

The present study results were represented with the help of tables, and charts. The results of scatter factor were compared between field size defined by jaws and MLC for both MLC design depicted in Table 1. The SF variation was $3.2 \%$ for lower field size in Mi-MLC and $2.4 \%$ for HD-MLC whereas for higher field size, it was reduced to $0.7 \%$ for Mi-MLC and $1.1 \%$ for HD-MLC. As shown in Table 2, the maximum SF difference was found in smaller field sizes on both MLC design.

The PDD was also measured for different field sizes defined by both MLC as well as jaws to analyse surface dose, dose at $10 \mathrm{~cm}$, depth of $90 \%$ and $80 \%$ dose and were noted down for both MLC design. As shown in Table 3, though results of PDD looks almost similar on both MLC design, the surface dose and was found to be slightly less in smaller field sizes and higher in larger field sizes in HD-MLC as compared to Mi-MLC. In addition, dose at $10 \mathrm{~cm}$ was slightly less in HD-MLC as compared to Mi-MLC. However, no significant dose difference was observed for the two MLC designs. Table 4 and Table 5 results revealed that depth of dose $80 \%$ and $90 \%$ were less in HD-MLC whereas for larger field sizes, depth of $80 \%$ dose is slightly higher in HD-MLC compared to Mi-MLC. The profiles were taken to quantify the symmetry, flatness and penumbra for field size $10 \times 10$ $\mathrm{cm}^{2}$ defined by MLC as well as jaws at depth of maximum dose (Dmax) was tabulated in Table 6 and Table 7 . The symmetry and flatness were observed within the limits. The results of penumbra were found to be less for HDMLC compared to Mi-MLC. A slight increase of MLC transmission factor and decrease of DLG were observed in Mi-MLC as compared to HD-MLC(Table 8). However, no significant difference was observed between two MLC models.

Table 1. Comparison of Scatter Factor between Mi-MLC and HD-MLC Model

\begin{tabular}{lcccccc}
\hline Field sizes $\left(\mathrm{cm}^{2}\right)$ & \multicolumn{2}{c}{ Mi-MLC } & \multicolumn{2}{c}{ Scatter factor (in water) } \\
& & & & HD-MLC \\
& MLC & Jaws & \% variation & MLC & Jaws & \% variation \\
\hline $2 \times 2$ & 0.812 & 0.787 & -3.2 & 0.803 & 0.784 & -2.4 \\
$5 \times 5$ & 0.914 & 0.898 & -1.9 & 0.913 & 0.894 & -2.1 \\
$10 \times 10$ & 1 & 1 & 0 & 1 & 1 & 0 \\
$20 \times 20$ & 1.085 & 1.093 & 0.73 & 1.09 & 1.102 & 1.1 \\
\hline
\end{tabular}

Table 2. Comparison of Scatter Factor for Field Size Defined by MLC between Two MLC Models

\begin{tabular}{lccc}
\hline Field Sizes $\left(\mathrm{cm}^{2}\right)$ & \multicolumn{3}{c}{ Scatter factor (Field defined by MLC) } \\
& Mi-MLC & HD-MLC & \% variation \\
\hline 2 X 2 & 0.812 & 0.803 & -0.01 \\
5 X 5 & 0.914 & 0.913 & -0.002 \\
$10 \times 10$ & 1 & 1 & 0 \\
20 X 20 & 1.085 & 1.09 & 0.005 \\
\hline
\end{tabular}

Table 3. Comparison of Surface Dose and Dose at $10 \mathrm{~cm}$ Depth for Different Field Sizes between Mi-MLC and HD-MLC

\begin{tabular}{lcccc}
\hline $\begin{array}{l}\text { Field size } \\
\left(\mathrm{cm}^{2}\right)\end{array}$ & \multicolumn{2}{c}{ Surface dose (\%) } & \multicolumn{2}{c}{ D10 cm (\%) } \\
\hline $2 \times 2$ & 47.04 & 48.31 & 59.35 & 59.31 \\
$5 \times 5$ & 49.85 & 50.23 & 62.85 & 62.81 \\
$10 \times 10$ & 54.24 & 54.82 & 66.73 & 66.32 \\
$20 \times 20$ & 63.05 & 62.38 & 69.54 & 69.5 \\
\hline
\end{tabular}

Asian Pacific Journal of Cancer Prevention, Vol $22 \mathbf{3 8 8 5}$ 
Table 4. Depth Dose Analysis of Truebeam STx for Field Sizes Defined by Jaws and HD MLC

\begin{tabular}{lllllllll}
\hline Field size $\left(\mathrm{cm}^{2}\right)$ & \multicolumn{2}{c}{ Surface dose $(\%)$} & \multicolumn{2}{c}{ Dose at $10 \mathrm{~cm}(\%)$} & \multicolumn{2}{c}{ Depth of 80\% $(\mathrm{mm})$} & \multicolumn{2}{c}{ Depth of 90\% (mm) } \\
& MLC & Jaws & MLC & Jaws & MLC & Jaws & MLC & Jaws \\
\hline $2 \times 2$ & 48.31 & 47.29 & 59.31 & 58.92 & 53.83 & 53.36 & 35.17 & 35.01 \\
$5 \times 5$ & 50.23 & 48.73 & 62.81 & 62.78 & 59.56 & 59.77 & 38.66 & 38.89 \\
$10 \times 10$ & 54.82 & 52.91 & 66.32 & 66.48 & 64.63 & 65.09 & 40.81 & 41.23 \\
$20 \times 20$ & 62.38 & 60.92 & 69.50 & 69.69 & 69.80 & 70.40 & 43.22 & 43.78 \\
\hline
\end{tabular}

Table 5. Depth Dose Analysis for Different Field Sizes Defined by Jaws and Mi-MLC

\begin{tabular}{lcccccccc}
\hline Field size $\left(\mathrm{cm}^{2}\right)$ & \multicolumn{2}{c}{ Surface dose $(\%)$} & \multicolumn{2}{c}{ Dose at $10 \mathrm{~cm}(\%)$} & \multicolumn{2}{c}{ Depth of 80\% (mm) } & \multicolumn{2}{c}{ Depth of 90\% $(\mathrm{mm})$} \\
& MLC & Jaws & MLC & Jaws & MLC & Jaws & MLC & Jaws \\
\hline $2 \times 2$ & 47.04 & 45.77 & 59.35 & 59.04 & 53.9 & 53.49 & 35.2 & 34.96 \\
$5 \times 5$ & 49.85 & 48.25 & 62.85 & 62.77 & 59.9 & 59.79 & 38.46 & 38.82 \\
$10 \times 10$ & 54.24 & 52.41 & 66.73 & 66.62 & 64.82 & 65.23 & 40.56 & 41.05 \\
$20 \times 20$ & 63.05 & 60.13 & 69.54 & 69.76 & 69.56 & 70.02 & 42.05 & 42.91 \\
\hline
\end{tabular}

\section{Discussion}

This study reveals that proper understanding and effect of dosimetric parameters vary with different MLC design from Varian linac before clinical treatment. During beam data measurements field size defined by both MLC and jaws have a significant role in beam modelling in the treatment planning system (TPS). There are few studies which have reported the dosimetric importance of different collimator designs from various linac systems.

The present study findings were supported by Mohan et al., (2008) who performed dosimetric characteristics of Varian 120 MLC using three different field-defining methods namely, jaw only, MLC only and MLC + jaw. The comparison results revealed that difference in the penumbra of $1.5 \mathrm{~cm}$ to $3.0 \mathrm{~cm}$ when compared between three methods in Mi-MLC wherein our study a difference of $0.53 \mathrm{~cm}$ to $0.49 \mathrm{~cm}$ and 0.38 to 0.25 in linac 1 and linac 2 for field size defined by MLC and jaw. Moreover, higher penumbra was found in Mi-MLC as compared HD-MLC for field size defined by MLC. Beyer (2013) compared

Table 6. Comparison of Cross Profile for Field Size Defined by Mi-MLC and HD-MLC

\begin{tabular}{|c|c|c|c|c|}
\hline \multirow{3}{*}{$\begin{array}{l}\text { MLC } \\
\text { model }\end{array}$} & \multicolumn{4}{|c|}{$10 \times 10 \mathrm{~cm}^{2}$ field size at $\mathrm{D}_{\max }$} \\
\hline & \multirow[t]{2}{*}{ Symmetry } & \multirow[t]{2}{*}{ Flatness } & \multicolumn{2}{|c|}{ Penumbra } \\
\hline & & & Right (mm) & Left (mm) \\
\hline Mi-MLC & 100.67 & 100.26 & 5.67 & 5.7 \\
\hline HD-MLC & 100.87 & 100.69 & 5.45 & 5.4 \\
\hline
\end{tabular}

Table 7. Comparison of Cross Profile for Field Defined by Jaw for Two Different Linac System

\begin{tabular}{lcccc}
\hline & \multicolumn{4}{c}{$10 \times 10 \mathrm{~cm}^{2}$ at $\mathrm{D}_{\max }$} \\
& Symmetry & Flatness & \multicolumn{2}{c}{ Penumbra $(\mathrm{mm})$} \\
& & & Right & Left \\
\hline Varian Unique & 100.63 & 100.38 & 5.14 & 5.21 \\
$\begin{array}{l}\text { Varian True } \\
\text { beam STx }\end{array}$ & 100.96 & 100.84 & 5.07 & 5.17 \\
\hline
\end{tabular}

Table 8. Comparison of MLC Transmission Factor and DLG for $6 \mathrm{MV}$ Photon Beam

\begin{tabular}{lcc}
\hline MLC model & MLC transmission factor & DLG (mm) \\
\hline HD-MLC & 0.011 & 0.318 \\
MI-MLC & 0.013 & 0.262 \\
\hline
\end{tabular}

measured photon beam data between three TrueBeam, Clinac 2100 and Trilogy linear accelerator to identify differences in the beam characteristics and to evaluate the possibility of beam matching for standard photon energies. Their results revealed that several similarities between Trilogy and Clinac 2100 and slight variation when compared to the TrueBeam linac. The difference could affect small field data and also large field sizes in beam matching considerations between the TrueBeam and previous linear accelerator models from the same manufacturer but should be within the accepted clinical tolerance for standard field sizes and standard treatments.

The scatter factors measured were in close agreement with Shende et al., (2016) measurements which is $0.2 \%$ and a significant variation was observed while comparing the same results with the study results of Tanaka et al., (2020) (1.2\%), Beyer (2013) and Chang et al., (2012) $(3.3 \%)$. This difference may be due to measurement set up Beyer (2013), and Chang et al., (2012) which measured output data at $5 \mathrm{~cm}$ depth whereas Shende et al., (2016), Tanaka et al., (2020) and our measurements were performed at $10 \mathrm{~cm}$ depth. The impact of the difference in measurement setup on scatter factor is another scope of study which is ongoing.

The dosimetric parameters such as surface dose, dose at $10 \mathrm{~cm}$ depth, depth of $80 \%$, depth of $90 \%$ were compared for both LINAC and both MLC from PDD measurements. Mohan et al., (2008) found a variation of $2 \%$ to $3 \%$ in surface dose when compared to MLC with that of MLC + jaws. Similarly, the present study revealed that the surface dose variation $2.8 \%$ to $4.9 \%$ between MLC and jaw for Linac 1 and $2.2 \%$ to $3.6 \%$ for linac 2 . A higher surface dose was found in MLC as compared to the jaws. Up to field size $10 \times 10 \mathrm{~cm}^{2}$ the surface dose 
was high for HD-MLC and above that surface dose is low for HD-MLC. This may be because the number of leaves to define the field is more for HD-MLC compared to Mi-MLC. There is an increased area of irradiation of MLC and hence resulted in the more scattered dose and resulted in higher surface dose whereas the dose at $10 \mathrm{~cm}$ depth was less than $0.4 \%$.

The penumbra from the cross profile which is the distance between $20 \%$ and $80 \%$ isodose at depth of maximum dose with fields defined by jaws are $5.5 \mathrm{~mm}$ to $5.7 \mathrm{~mm}$ for TrueBeam and $5.4 \mathrm{~mm}$ for Clinac and $5.5 \mathrm{~mm}$ for Trilogy machine as per Beyer (2013) whereas Shende et al., (2016) found that $5.5 \mathrm{~cm}$ (left) and $5.0 \mathrm{~cm}$ (right) for Truebeam. The present study found that a penumbra of $5.07 \mathrm{~mm}$ and $5.17 \mathrm{~mm}$ for Truebeam and $5.14 \mathrm{~cm}$ and $5.21 \mathrm{~cm}$ for Unique.

The DLG is the parameter to model the round leaf end effect of MLC and also an important parameter for the accurate calculation of IMRT and arc therapy plans. As per Lin et al., (2018) MLC system with less scattering and transmitting radiation should also have a smaller DLG value. From the penumbra values, it was evident that the Mi-MLC shows a slow dose fall off and hence the DLG value is less for Mi-MLC which is clear from our values.

This study shows the understanding of different dosimetric parameters between jaws and MLC which are necessary to evaluate at the time of commissioning and before clinical implementation. Though the planning system requires the data with field sizes defined by jaws, it is also necessary to check the same data with MLC for selected field size if not for all field sizes and it should be comparable. From the overall analysis, the study results concluded that both the MLC designs have merits and demerits that are based on their effectiveness and clinical use. However, the higher surface dose was found in HD-MLC in contrast to Mi-MLC.

\section{Author Contribution Statement}

The authors confirm contribution to the paper as follows: study conception and design: Aswathi Raj, data collection, analysis and interpretation of results: Aswathi Raj and Hridya V T; draft manuscript preparation: Aswathi Raj. All authors reviewed the results and approved the final version of the manuscript.

\section{Acknowledgments}

None.

\section{Statement conflict of Interest}

$\mathrm{I} /$ we have no financial interest in any of the technologies used in this study. We didn't get any kind of grant or financial support from any organization.

\section{References}

AAPM Radiation Therapy Committee, Boyer A (2001). Basic applications of multileaf collimators. Medical Physics Publishing.

Alipour H, Hadad K, Faghihi R (2019). Investigation of physical penumbra definition in treatment planning. Int J Radiat Res, 17, 493-7.

Asnaashari K, Chow JC, Heydarian M (2013). Dosimetric comparison between two MLC systems commonly used for stereotactic radiosurgery and radiotherapy: a Monte Carlo and experimental study. Physica Med, 29, 350-6.

Beyer GP (2013). Commissioning measurements for photon beam data on three TrueBeam linear accelerators, and comparison with Trilogy and Clinac 2100 linear accelerators. J Appl Clin Med Phys, 14, 273-88.

Chang Z, Wu Q, Adamson J, et al (2012). Commissioning and dosimetric characteristics of TrueBeam system: composite data of three TrueBeam machines. Med phys, 39, 6981-7018.

ChowJC, Seguin M, Alexander A (2005). Dosimetric effect of collimating jaws for small multileaf collimated fields. Med Phys, 32, 759-65.

Galvin James M (1999). The multileaf collimator: a complete guide." In Proc. AAPM annual meeting.

Glide-Hurst C, Bellon M, Foster R, et al(2013)“Commissioning of the Varian TrueBeam linear accelerator: a multiinstitutional study. Med Phys, 40, 031719.

Godson HF, Ravikumar M, Sathiyan S, et al (2016). Analysis of small field percent depth dose and profiles: Comparison of measurements with various detectors and effects of detector orientation with different jaw settings. J Med Phys Assoc Med Phys India, 41, 12.

Huq MS, Das I J, d Steinberg T, Galvin JM (2002) A dosimetric comparison of various multileaf collimators. Phys Med Biol, 47, N159.

Jeraj M, Robar V (2004). Multileaf collimator in radiotherapy. Radiol Oncol, 38.

Kehwar TS, Bhardwaj AK, Chakarvarti SK (2006). Evaluation of dosimetric effect of leaf position in a radiation field of an 80-leaf multileaf collimator fitted to the LINAC head as a tertiary collimator. J Appl Clin Med Phys, 7, 43-54.

Lin CY, Shiau AC, Ji JH, et al (2018). A simple method for determining dosimetric leaf gap with cross-field dose width for rounded leaf-end multileaf collimator systems. Radiat Oncol, 13, 1-7.

Mahmoudi A, Ghazale G, Alireza S. (2019) "Penumbra reduction technique and factors affecting it in radiotherapy machinesReview study. Radiat Phys Chem, 157, 22-7.

Omar MK, Khaled ME, Eldebawi NM, Mansour NA (2013) Dosimetric evaluation of the Multileaf collimator for irregular shaped radiation fields. Indian J Appl Res, 3.

Park SY, Siyong K, Wonmo S, Sang-TK (2019) Modeling scattered radiation from multi-leaf collimators (MLCs) to improve calculation accuracy of in-air output ratio. Australasian Phys Eng Sci Med, 42, 719-31.

Mohan R,Jayesh K, Joshi RC, et al (2008). Dosimetric evaluation of 120-leaf multileaf collimator in a Varian linear accelerator with 6-MV and 18-MV photon beams. $J$ Med Phys, 33, 114-8.

Sharma D, Dongre P, Mhatre V, Heigrujam M (2017). Physical and dosimetric characteristic of high-definition multileaf collimator (HDMLC) for SRS and IMRT. J Appl Clin Med Phys, 12, 142-60.

Shende R, Gupta G, Patel G, Kumar S (2016). Commissioning of TrueBeam TM medical linear accelerator: quantitative and qualitative dosimetric analysis and comparison of flattening filter (FF) and FLATTENING FILTER FRee (FFF) beam. Int J Med Phys Clin Eng Radiat Oncol, 5, 51-69.

Tanaka Y, Mizuno H, AkinoY, et al (2020). Collection and analysis of photon beam data for Varian C-series linear accelerators: a potential reference beam data set. Phys Eng Sci Med, 43, 889-901. 
Aswathi Raj et al

\section{(c) (i) (8)}

This work is licensed under a Creative Commons Attribution-

Non Commercial 4.0 International License. 